\title{
Associations between adherence, depressive symptoms and health-related quality of life in young adults with cystic fibrosis
}

\author{
K. B. Knudsen ${ }^{* *}$, T. Pressler ${ }^{2}$, L. H. Mortensen ${ }^{3}$, M. Jarden ${ }^{3,4}$, M. Skov², A. L. Quittner ${ }^{5}$, T. Katzenstein ${ }^{1}$ \\ and K. A. Boisen ${ }^{6}$
}

\begin{abstract}
Background: Cystic fibrosis (CF) is a life shortening disease, however prognosis has improved and the adult population is growing. Most adults with cystic fibrosis live independent lives and balance the demands of work and family life with a significant treatment burden. The aim of this study was to examine the relationships among treatment adherence, symptoms of depression and health-related quality of life (HRQoL) in a population of young adults with CF.

Methods: We administered three standardized questionnaires to 67 patients with CF aged 18-30 years; Morisky Medication Adherence Scale, Major Depression Inventory, and Cystic Fibrosis Questionnaire-Revised.

Results: There was a response rate of $77 \%$ and a majority of the young adults ( $84 \%$ ) were employed or in an education program. Most participants (74\%) reported low adherence to medications. One third (32.8\%) of the participants reported symptoms of depression. HRQoL scores were especially low on Vitality and Treatment Burden, and symptoms of depression were associated with low HRQoL scores $(p<0.01)$ with medium to large deficits across on all HRQoL domains (Cohen's d 0.60-1.72) except for the domain treatment burden. High depression symptom scores were associated with low adherence $(r=-0.412, p<0.001)$.

Conclusions: Despite improved physical health, many patients with CF report poor adherence, as well as impaired mental wellbeing and HRQoL. Thus, more attention to mental health issues is needed.
\end{abstract}

Keywords: Cystic fibrosis, Adherence, Depression, Quality of life, Mental health, Young adults

\section{Background}

Cystic fibrosis (CF) is a genetic, chronic and fatal disease that is often diagnosed early in life. CF is a multi-organ disease which primarily affects the pancreas, digestive and pulmonary systems, leading to risk of diabetes, malnutrition and chronic pulmonary infections (Spoonhower and Davis 2016). From the time of diagnosis until death, the treatment regimen is a substantial part of daily life. This regimen includes chest physiotherapy, inhalation treatments, oral and intravenous medications. Some

\footnotetext{
*Correspondence: Karin.baek.knudsen.01@regionh.dk

1 The Department of Infectious Diseases, Copenhagen University Hospital, Rigshospitalet, Blegdamsvej 9, 2100 Copenhagen, Denmark

Full list of author information is available at the end of the article
}

of the treatments are daily and preventive, while others are therapeutic and may be increased during pulmonary exacerbations (O'Sullivan and Freedman 2009). Optimization of medical treatment has improved survival, however respiratory failure is the main cause of death. Thirty years ago, few individuals with CF reached adulthood. Today, the average life expectancy for newborns with CF is approximately 40 years and is projected to be more than 50 years if mortality continues to decrease at the rate observed between 2000 and 2010 (MacKenzie et al. 2014; Ratjen et al. 2015). As a result, there is an increased focus on adolescents' transition into adulthood, as well as issues of adherence, mental health and healthrelated quality of life (HRQoL) (Quittner et al. 2014a, b; Sawicki et al. 2011). In qualitative studies, young adults 
have described their lives with CF as a complex balance between leading a healthy normal life and feeling different from peers due to the burdensome aspects of disease management (Jessup and Parkinson 2010; Badlan 2006). Many find it challenging to integrate the prescribed treatments into their busy lives, and this has been found to affect psychological well-being and HRQoL (Sawicki and Goss 2015).

Recently, studies have found poor adherence (Quittner et al. 2014a) and a high prevalence of depression in CF populations (Quittner et al. 2014b; Quittner et al. 2008; Modi et al. 2011). However, both rates of adherence and depression vary between different patient populations and few studies have included the Danish $\mathrm{CF}$ population. There is currently no gold standard for measuring adherence to prescribed medications, and rates of adherence also vary considerably depending on how adherence is measured (Modi et al. 2006). The International Depression/Anxiety Epidemiological Study of CF patients (TIDES), conducted at 154 CF centers in nine countries in Europe and USA, reported elevated rates of depression for adults across countries, according to age, screening measure, and country (Quittner et al. 2014b). Overall, $10 \%$ of adolescents and $19 \%$ of adults had elevated depression scores, depending on the screening tool. The Hospital Anxiety and Depression scale (HADS) yielded the lowest scores (11\% elevated) versus 27 \% elevated on the Center for Epidemiological Studies Depression Scale (CES-D) (Quittner et al. 2014b). Note however, that HADS has been criticized both for a lack of sensitivity and a failure to represent the major symptoms underlying depression diagnosed using the DSMIV or ICD-10 (Cameron et al. 2008). Denmark was not included in TIDES study and thus, the prevalence of depression among Danish adolescents and adults with $\mathrm{CF}$ is not known. It was hypothesized that adults with CF who had symptoms of depression would have lower rates of adherence and impaired HRQoL. The aim of this study was to examine rates of adherence to prescribed treatments, assess symptoms of depression, evaluate HRQoL, and test the associations between adherence, depression and HRQoL in young adults with CF.

\section{Methods}

\section{Material and participants}

This was a cross-sectional study using well-validated questionnaires administered to young adults aged $18-30$ years with a confirmed diagnosis of cystic fibrosis (CF). Participants were recruited from November 2013 to December 2014 at a specialized, outpatient clinic for $\mathrm{CF}$ at the Copenhagen University Hospital, Rigshospitalet, which is one of the two CF centers in Denmark. The Danish CF population consists of 460 people and about 300 are followed at Rigshospitalet. Participants were recruited during their regular outpatient visit, were informed about the study and gave their oral informed consent to participate by completing the questionnaires. For patients who did not attend the outpatient clinic regularly, questionnaires were sent by mail with a selfaddressed, stamped return envelope. The questionnaires were provided with an explanatory letter indicating that a final consent was inferred from completing and returning the questionnaire.

Data on demographic and clinical characteristics were extracted from the Danish CF Registry for the entire CF population at Rigshospitalet aged $18-30$ years, and are described as the mean of all measurements obtained in 2014. Results from lung function tests were obtained via chart review, measured by forced expiratory volume in one second $\left(\mathrm{FEV}_{1}\right)$. $\mathrm{FEV}_{1}$ values in liters were transformed into the percent of predicted $\left(\mathrm{FEV}_{1} \%\right)$ as described by Hankinson et al. (1999).

\section{Patient-reported outcome measures}

Three questionnaires were included and filled out at one time point: Morisky Medication Adherence Scale (MMAS-8) (Morisky et al. 2008) is a validated self-report questionnaire that measures adherence to prescribed medications, includes specific medication-taking behaviors, and identifies common barriers to poor adherence. It is an 8 item test, 7 items rated dichotomously as "yes/ no" responses ( 0 or 1 point), and one item rated on a 5 -point frequency scale from 0 (never or rarely), 0.25 (once in a while), 0.50 (sometimes), 0.75 (usually) to 1 (always) point. A total score on the MMAS ranges from 0 to 8 points; $<6$ point indicates low adherence, $6<8$ points indicates medium adherence and 8 points reflects high adherence (Morisky et al. 2008). MMAS-8 has been translated to several languages and many psychometric tests of the translated instrument have been performed. The scale has demonstrated good sensitivity but only moderate internal consistency. The scale has also demonstrated convergent validity with electronic measures and is a reliable tool to determine medication adherence (Morisky et al. 2008; Arnet et al. 2015).

The Major Depression Inventory (MDI) (Bech et al. 2001) is a self-administered instrument that measures depressive symptoms. It consists of 10 items that assess the ICD-10 and DSM-IV (WHO 1993; APA 1994) symptoms of major depressive illness. On a 6-point Likert scale, individual items measure how much of the time these symptoms have been present during the past $24 \mathrm{~h}$. The scale ranges from 0 (symptoms have not been present at all) to 5 (symptoms have been present all of the time). To fulfill the ICD-10 criteria of moderate to severe depression, at least two of the three core symptoms need 
to be present. The three core symptoms are: depressed mood, lack of interest, and lack of energy. Scores range from 0 to 50; categorized into 'No depression' (0-19 points), 'Mild depression' (20-24 points), 'Moderate depression' (25-29 points), 'Severe depression' (more than 29 points) (Bech et al. 2001). Sensitivity and specificity for DSM-IV has been found to be high for the MDI (Olsen et al. 2003). A recent psychometric evaluation of the MDI as a depression severity scale confirmed these cut-off scores (Bech et al. 2015). Participants screened positive for moderate-severe depression, with a MDI score $>24$, were contacted and offered a consultation with a psychologist.

The Cystic Fibrosis Questionnaire-Revised-Teen/Adult version (CFQ-R) (Quittner et al. 2012; Bregnballe et al. 2008) is a well-validated, disease-specific HRQoL instrument for individuals with CF ages 14 years and older. It has undergone extensive reliability and validity testing and is now considered the "gold standard" measure of HRQoL for CF. The CFQ-R assesses demographic information (e.g., age, education) and measures twelve domains of functioning: Physical Functioning, Vitality, Emotional Functioning, Eating Disturbances, Treatment Burden, Health Perceptions, Social Functioning, Body Image, Role Functioning, Weight, Respiratory Symptoms and Digestive Symptoms. Scores are standardized and range from 0 to 100 , with higher scores indicating better quality of life (Quittner et al. 2012).

\section{Statistical analysis}

Means and standard deviations were calculated for MMAS-8, MDI and CFQ-R, as well as demographic and clinical characteristics. Independent $\mathrm{t}$-tests were used to determine if there was a difference between participants and non-participants. To test how the CFQ-R was associated with other characteristics, a one-way multivariate analysis of variance (MANOVA) was used to test overall differences.

MANOVAs were carried out to test whether independent variables, such as gender, age group (18-23 years/2430 years), marital status (single/partner), level of education (lower education/high school/college or university), work/education ability versus work/education disability, adherence (MMAS score $<6 /$ MMAS score $\geq 6$ ) and depression (MDI score $<20$ /MDI score $\geq 20$ ) were associated with the twelve domains of CFQ-R. To control for increasing Type I error rate, statistically significant MANOVAs were followed up by univariate ANOVAs to test domain-specific differences. To estimate the magnitude of the association between symptoms of depression, ability to work and HRQoL, effect sizes were calculated by subtracting the means and dividing the result by the pooled standard deviation, resulting in Cohen's d. Given the variable and small sample sizes, calculation of the pooled standard deviation was adjusted with weight for sample sizes according to Hedges and Olkin (Lakens 2013).

To determine the strength of the linear relationship between MMAS-8 and MDI, we calculated a Pearson correlation coefficient. Logistic regression was performed to ascertain the effects of gender, age, civil status, level of education, work/education ability related to health on adherence (MMAS score $<6$ /MMAS score $\geq 6$ ) and depression (MDI score $<20 / M D I$ score $\geq 20$ ), respectively. Data management and analysis were performed using IBM SPSS Statistics version 20. A p value $<0.05$ was considered statistically significant.

\section{Results}

During the study period 90 patients aged $18-30$ years were registered at the Copenhagen CF Center with a confirmed CF diagnosis. Three patients lived abroad during the study period and were excluded. Among the 87 eligible patients, 67 completed the questionnaires (response rate $77 \%$ ). These measures were completed at the outpatient clinic by 61 patients; however 12 patients who did not attend the clinic regularly received the questionnaires by mail, and six of these patients returned the completed questionnaires. In total, 20 patients did not fill in the questionnaires, four refused to participate and 16 did not respond to inquiries. No significant differences were found between the participants versus non-participants on any demographic characteristics (see Table 1). Most participants (84 \%) were employed or in an education program and $3 \%$ were unemployed. Nine participants $(13 \%)$ were unable to work or study due to health problems.

\section{Adherence}

MMAS- 8 was completed by 66 participants. Forty-nine $(74.2 \%)$ scored in the low adherence range (MMAS score $<6), 12(18.2 \%)$ had medium adherence (MMAS score $6<8)$, and five $(7.6 \%)$ reported high adherence (MMAS score $=8)($ Table 2$)$.

\section{Depression}

The MDI was completed by all participants. MDI scores above 20 indicated symptoms of depression and were endorsed by $32.8 \%$ of the participants; $19.4 \%$ expressed symptoms of moderate or severe depression (Table 2); of these, $77 \%$ had consulted a psychologist or psychiatrist and/or received antidepressant medication. The three remaining participants were contacted and offered a consultation with a psychologist, but all claimed to be feeling well, and had been sad or worried the day they completed the questionnaires. Females were more likely 
Table 1 Demographic and clinical characteristics of participants

\begin{tabular}{|c|c|c|c|}
\hline & Study participants & Non-participants & $p$ value \\
\hline Number & 67 & 20 & \\
\hline Age mean (range) & $24.1(18-30)$ & $22.6(18-30)$ & 0.09 \\
\hline Females, n (\%) & $38(59)$ & $8(40)$ & 0.20 \\
\hline $\begin{array}{l}\text { BMI }\left(\mathrm{kg} \mathrm{m}^{2)} \text {, mean }\right. \\
\text { (SD) }\end{array}$ & $21.8(3.6)$ & $21.2(2.2)$ & 0.40 \\
\hline $\begin{array}{l}\text { FEV1 \% predicted, } \\
\text { mean (SD) }\end{array}$ & $72.2(23)$ & $74(22)$ & 0.80 \\
\hline FEV1 ( $\leq 40 \%), n(\%)$ & $7(11)$ & $1(5)$ & \\
\hline FEV1 (41-70 \%), n (\%) & $25(38)$ & $7(35)$ & \\
\hline FEV1 (>71 \%), n (\%) & $33(51)$ & $12(60)$ & 0.70 \\
\hline $\begin{array}{l}\text { Chronic pulmonary } \\
\text { infectiona }, n(\%)\end{array}$ & $40(62)$ & $10(50)$ & 0.30 \\
\hline CFRD, $n(\%)$ & $18(28)$ & $7(35)$ & 0.40 \\
\hline Single $e^{b}, n(\%)$ & $33(49)$ & NA & \\
\hline $\begin{array}{l}\text { Lower education, } \\
n(\%)\end{array}$ & $21(31)$ & NA & \\
\hline High school, $n(\%)$ & $18(27)$ & NA & \\
\hline $\begin{array}{l}\text { College/university, } \\
n(\%)\end{array}$ & $19(28)$ & NA & \\
\hline $\begin{array}{l}\text { Employed or study- } \\
\text { ing, } n(\%)\end{array}$ & $56(84)$ & NA & \\
\hline $\begin{array}{l}\text { Incapacitated to } \\
\text { work/study, n (\%) }\end{array}$ & $9(13)$ & NA & \\
\hline
\end{tabular}

CFRD cystic fibrosis related diabetes

a Pseudomonas aeruginosa, Achromobacter xylosoxidans and Burkholderia species

b Single status is self-reported and defined as not having a romantic partner

Table 2 Distribution of MDI and MMAS scores

\begin{tabular}{lc}
\hline & N (\%) \\
\hline No depression (MDI score 0-19) & $45(67.2)$ \\
Mild depression (MDI score 20-24) & $9(13.4)$ \\
Moderate depression (MDI score 25-29) & $5(7.5)$ \\
Severe depression (MDI score 30-50) & $8(11.9)$ \\
Low adherence (MMAS score $<6)$ & $49(74.2)$ \\
Medium adherence (MMAS score $=6<8)$ & $12(18.2)$ \\
High adherence (MMAS score $=8)$ & $5(7.6)$ \\
\hline
\end{tabular}

to report more symptoms of depression on the MDI; with a female/male OR of 5.1 (95\% CI 1.03-25.3) for moderate/severe depression scores.

\section{Quality of life}

The CFQ- $R$ scores across the 12 domains of functioning are displayed in Table 3. Vitality and Treatment Burden were the lowest scores (47.1-52.9), indicating poor HRQoL; in Body Image and Eating Disturbances were the highest scores (77.8-83.4).
Associations between adherence, depression and quality of life

To test how the CFQ-R was associated with independent variables, such as gender, age, civil status, level of education, work/education ability, adherence and depression, a one-way multivariate analysis of variance (MANOVA) was used to test overall differences. There were statistically significant difference between CFQ-R and depressive symptoms $(\mathrm{p}<0.01)$ and work/education ability $(\mathrm{p}<0.001)$. Thus, one way ANOVA analyses of the main effect of depressive symptoms and work/education ability were performed and result of the effects on the twelve domains are shown in Table 3. Participants who reported depression and work/education disability scored significantly lower on all domains of the CFQ-R, except Treatment Burden. We found medium to large effects for these associations. We found significantly higher HRQoL scores for those who had a MDI score $<19$ ('No depression') compared to those who had a MDI score $20<24$ ('Mild depression'), with similar results for Physical Functioning $(\mathrm{p}=0.048)$, Role Limitations $(\mathrm{p}=0.009)$, Vitality $(p=0.003)$, Emotional functioning $(p=0.001)$, Social Functioning $(\mathrm{p}=0.02)$, Health Perception $(\mathrm{p}=0.001)$ and Respiratory Symptoms $(\mathrm{p}=0.03)$. No associations were found between the MMAS-8 scores and the CFQ-R.

Depression and adherence scores were negatively associated, increases in MDI scores were associated with decreases in MMAS scores (see Fig. 1), indicating that symptoms of depression were associated with worse adherence, $\mathrm{r}=-0.412, \mathrm{p}<0.001$. Pearson correlations were performed with and without the outlier, with no difference in the results.

An increase in MDI scores was moderately correlated with a decrease in MMAS scores, $r=-0.412, p<0.001$.

\section{Discussion}

In this cross sectional study, which examined rates of adherence and depression in relation to HRQoL, we found a large number of young adults with CF who reported poor adherence, high rates of depression and impaired HRQoL.

The MMAS-8 was a useful screening tool, identifying a majority of participants with low adherence (74\%). In a large national US study, both pediatric and adult patients with CF picked up $50 \%$ or fewer prescribed medications (Quittner et al. 2014a). A recent systematic review found generally poor adherence in patients with $\mathrm{CF}$, with a lack of consensus on how to measure adherence (O'Donohoe and Fullen 2014). Generally higher rates of adherence have been found in studies using self-report measures in comparison to more objective measures (e.g., medication possession ratios, electronic measures) (Modi et al. 2006). The MMAS- 8 is short (8 questions), easy to 
Table 3 CFQ-R domain scores in relation to depression scores and employability

\begin{tabular}{|c|c|c|c|c|c|c|c|c|c|}
\hline & \multirow{2}{*}{$\begin{array}{l}\text { Total }(\mathrm{N}=67) \\
\text { Mean (SD) }\end{array}$} & \multirow{2}{*}{$\begin{array}{l}\text { No depression } \\
(\mathrm{N}=45) \\
\text { Mean (SD) }\end{array}$} & \multirow{2}{*}{$\begin{array}{l}\text { Symptoms } \\
\text { of depression } \\
(\mathrm{N}=22) \\
\text { Mean (SD) }\end{array}$} & \multicolumn{2}{|c|}{$\begin{array}{l}\text { No depression ver- } \\
\text { sus depression symp- } \\
\text { toms }\end{array}$} & \multirow{2}{*}{$\begin{array}{l}\text { Work/educa- } \\
\text { tion ability } \\
(\mathrm{N}=58) \\
\text { Mean (SD) }\end{array}$} & \multirow{2}{*}{$\begin{array}{l}\text { Work/educa- } \\
\text { tion disability } \\
(\mathrm{N}=9) \\
\text { Mean (SD) }\end{array}$} & \multicolumn{2}{|c|}{$\begin{array}{l}\text { Work/education ability } \\
\text { versus disability }\end{array}$} \\
\hline & & & & Effect size ${ }^{a} d$ & p value ${ }^{b}$ & & & Effect size $^{a} d$ & $p_{\text {value }}$ b \\
\hline $\begin{array}{l}\text { Physical func- } \\
\text { tioning }\end{array}$ & $72.8(27.3)$ & 80.7 (22.6) & $56.5(28.9)$ & -0.98 & 0.001 & $79.3(21.8)$ & $31.0(22.0)$ & -2.21 & 0.001 \\
\hline $\begin{array}{l}\text { Role limita- } \\
\text { tions }\end{array}$ & 73.5 (26.0) & $81.7(21.2)$ & $52.6(27.0)$ & -1.25 & $<0.001$ & 80.6 (18.2) & $22.6(12.4)$ & -3.09 & 0.001 \\
\hline Vitality & $47.1(25.3)$ & $58.1(21.2)$ & $26.4(15.5)$ & -1.62 & $<0.001$ & $50.2(24.4)$ & $27.2(22.3)$ & -0.93 & 0.01 \\
\hline $\begin{array}{l}\text { Emotional } \\
\text { functioning }\end{array}$ & $69.3(20.8)$ & $78.4(13.8)$ & $50.0(21.1)$ & -1.72 & $<0.001$ & $73.0(18.7)$ & $45.2(17.6)$ & -1.50 & 0.001 \\
\hline $\begin{array}{l}\text { Social func- } \\
\text { tioning }\end{array}$ & $70.8(20.7)$ & $77.0(19.2)$ & $57.7(14.4)$ & -1.10 & $<0.001$ & $73.2(19.7)$ & $55.4(21.2)$ & -0.90 & 0.02 \\
\hline Body image & $77.8(23.3)$ & $84.8(17.1)$ & $61.1(27.8)$ & -1.12 & $<0.001$ & $81.3(20.7)$ & $55.6(27.8)$ & -1.18 & 0.002 \\
\hline $\begin{array}{l}\text { Eating distur- } \\
\text { bances }\end{array}$ & $83.4(25.2)$ & 89.4 (20.6) & $70.4(31.7)$ & -0.77 & 0.007 & $85.6(24.1)$ & $69.1(28.7)$ & -0.67 & 0.07 \\
\hline $\begin{array}{l}\text { Treatment } \\
\text { burden }\end{array}$ & $52.9(21.8)$ & $56.1(21.7)$ & $47.5(22.5)$ & -0.39 & 0.17 & $54.0(21.2)$ & $45.7(25.7)$ & -0.38 & 0.3 \\
\hline $\begin{array}{l}\text { Health percep- } \\
\text { tion }\end{array}$ & $55.2(29.1)$ & $67.7(23.7)$ & $29.0(22.9)$ & -1.65 & $<0.001$ & $60.0(27.6)$ & $24.7(19.1)$ & -1.21 & 0.001 \\
\hline $\begin{array}{l}\text { Weight prob- } \\
\text { lems }\end{array}$ & $72.2(38.6)$ & $78.3(34.8)$ & $55.6(42.8)$ & -0.60 & 0.04 & 79.5 (32.6) & $25.9(43.4)$ & -1.68 & 0.001 \\
\hline $\begin{array}{l}\text { Respiratory } \\
\text { symptoms }\end{array}$ & $62.9(25.0)$ & $69.5(21.5)$ & $50.0(26.5)$ & -0.84 & 0.008 & $65.8(23.7)$ & $45.1(27.0)$ & -0.76 & 0.02 \\
\hline $\begin{array}{l}\text { Digestive } \\
\text { symptoms }\end{array}$ & $74.0(24.4)$ & $80.1(21.2)$ & $62.3(27.0)$ & -0.77 & 0.03 & $73.8(25.1)$ & $75.3(20.6)$ & 0.06 & 0.9 \\
\hline
\end{tabular}

${ }^{a}$ Cohen's $d$ corrected effect sizes are classified as small $(d=0.2)$, medium $(d=0.5)$, large $(d=0.8)$ and very large $(d=1.3)^{b}$ Values in italic represent $p<0.05$

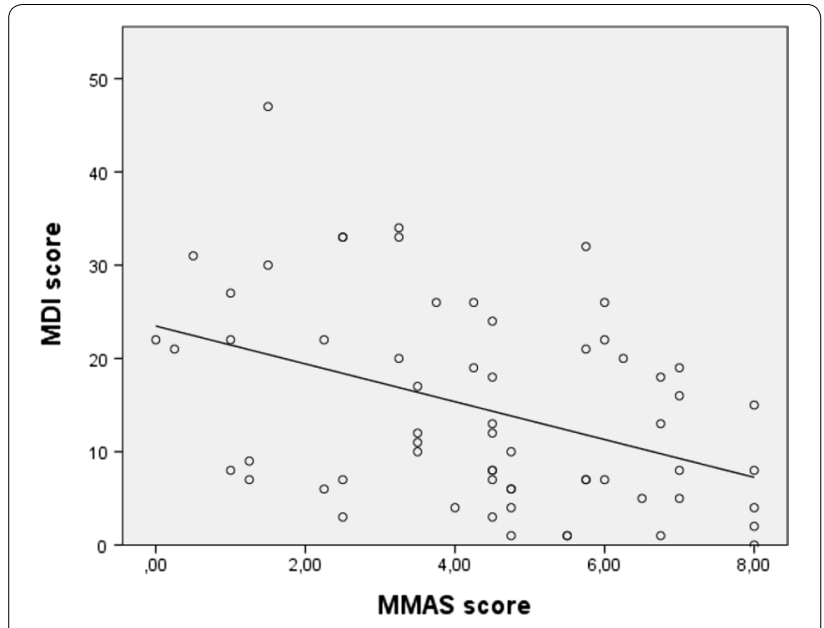

Fig. 1 Correlation between depression scores and adherence scores

understand, and adaptable for different kinds of medications (Culig and Leppee 2014). According to the WHO, non-adherence among patients with chronic diseases is about $50 \%$ in developed countries (Sabaté 2003). Several studies have suggested that barriers to CF treatment adherence include lack of time, forgetting, social stigma, and treatment burden (George et al. 2010; Bregnballe et al. 2011). Treatment complexity in CF has increased over the past decades and high treatment complexity scores have been inversely related to Treatment Burden on the CFQ-R, indicating that increasing complexity is associated with worse perceptions of burden (Sawicki et al. 2013). In a qualitative study, patients reported that it was a challenge to fit these time-consuming treatments into daily life, and some patients were intentionally nonadherent to permit spontaneity, independence and social activities (George et al. 2010).

In the current study, we found low scores on the CFQ-R Treatment Burden domain, indicating high perceptions of treatment burden across patients, and this effect was independent of work ability or symptoms of depression. We found a moderate negative correlation between depression and adherence to treatment. Fidika et al. (2014) reported a decline in lung function (FEV1 \% predicted) in adolescents and adults with CF with elevated symptoms of depression in a 2-year observation study. Our results support their hypothesis that depressed patients may fail to perform their prescribed treatments due to mental health problems, such as depression. Thus, our findings suggest that treating depression is an important target for intervention, and may have positive effects 
on lung function and other health outcomes. In addition, high perceptions of treatment burden and poor adherence should be addressed using systematic, behavioral interventions. Brief, behavioral interventions have been shown to be most effective in individuals with chronic conditions (e.g., HIV) (Gross et al. 2013). These interventions should be tested in the Danish CF population.

In our study, one third of the participants screened positive for depression and $19.4 \%$ were in the moderate to severe range. These Danish results are similar to the highest rates reported in TIDES study, with rates of 29 \% reported at centers using the CES-D as a screening tool (Quittner et al. 2014b). In our study, we used the MDI, a screening tool used to analyze the prevalence of depression in the general population (Olsen et al. 2003). Good sensitivity and specificity, as well as responsiveness to antidepressant treatments, have been reported for the MDI (Bech et al. 2001; Konstantinidis et al. 2011). The MDI was not developed for patients with CF and there is a risk of identifying false positives. However, it is worth noting that the group of participants who had symptoms of mild depression, compared to those with no symptoms of depression, scored significantly lower on HRQoL domains that measure psychosocial functioning. Thus, even if the group of patients with mild depression symptoms is overestimated, it could be an indication of a need for more psychosocial support. These findings are in agreement with the consensus statements from The International Committee on Mental Health in Cystic Fibrosis which recommended that adolescents and adults with CF whose depression or anxiety is in the mild range should be provided with information about depression and anxiety and receive preventive or supportive psychological interventions (Quittner et al. 2016). All clinically elevated scores from screening tools should be followed up with a more in-depth clinical interview. Importantly, $77 \%$ of the 13 participants who reported moderate or severe symptoms of depression had already consulted a psychologist or psychiatrist and/or received antidepressant medication, suggesting that using the MDI as a screener provided valid data. Unfortunately, we have no information on the length or outcomes of these psychological or pharmacological treatments. Given that their depressive symptoms have persisted, it may be important to evaluate evidence-based psychological interventions, tailored specifically to the needs of those with $\mathrm{CF}$ (Quittner et al. 2016). Current evidence for psychological interventions is insufficient, but cognitive behavioral therapy have shown the most promising results according to a recent Cochrane review by Goldbeck et al. 2014.

Comparing the CFQ-R results of this study with the national, normative data from the US Epidemiological Study of CF (ESCF) which had 4679 participants (ages $\geq 14$ years) (Quittner et al. 2012), our scores on all domains, except Physical Functioning, Body Image and Weight were lower for this cohort of young Danish adults. The scores in this study are comparable to those reported in a former Danish CF study of patients with moderately reduced lung function $\left(\mathrm{FEV}_{1} 41-70 \%\right.$ predicted) (Bregnballe et al. 2008), however more than $51 \%$ of the participants in our study had a lung function value greater than $70 \%$ of predicted. This study confirms the results of prior studies which have found that depression is related to worse HRQoL on the CFQ-R. (Havermans et al. 2008; Riekert et al. 2007; Yohannes et al. 2012).

Young adults are likely still transitioning to adult roles, including starting a career and family, which according to Chick and Meleis (1986) may be characterized by disorientation, distress, irritability, anxiety and depression. In our study, we found markedly reduced HRQoL among participants who were not studying or working due to health problems. A link between worse lung function and reduced HRQoL scores among individuals with CF was described by Riekert et al. (2007), however, lung function alone does not reflect an accurate picture of a patient's working capabilities (Frangolias et al. 2003), and in a previous study, decreased work capacity was correlated with HRQoL, but not with lung function (Targett et al. 2014). Having a job clearly has positive effects, such as improved financial prosperity, social participation, selfesteem and psychological well-being (Targett et al. 2014). Thus, a combination of inability to work and increasing health problems negatively impact HRQoL.

This study includes the majority of young adults with $\mathrm{CF}$ in Copenhagen, with a high response rate. The Copenhagen CF population is generally in a state of good health, with relatively high lung function and BMI, despite severe CF-mutations (Knudsen et al. 2015). Treatment of Danish patients with CF has been centralized since 1962, and these patients are followed at the CF center every month and monitored closely. The limitations of this study include the relatively small sample size and the use of questionnaires that have not been validated in a CF population. The Danish translation of MMAS- 8 was conducted with a great deal of rigor, but it has not been validated in a psychometric study. MMAS- 8 measures 'total' adherence, thus differences in adherence between types of treatment cannot be assessed. The MDI is validated and widely used in Denmark in the general population; however it is not a common screening tool for depression worldwide. The International Consensus Guidelines on Mental Health in CF recommended using the PHQ-9 and GAD-7 to screen patients with CF each year, beginning at age 12 (Quittner et al. 2016). According to Danish law, questionnaire studies can be done without the participants' written consent; however, this implies 
that individual health data cannot be obtained. Consequently, it was not possible to investigate how $\mathrm{FEV}_{1}$ correlated with work disability and HRQOL.

\section{Conclusions}

Our study demonstrated that among young adults with $\mathrm{CF}$, adherence to prescribed treatments is low, symptoms of depression are prevalent and quality of life is impaired. Our study confirms that symptoms of depression have a significant and negative effect on HRQOL. Finally, we demonstrated that the MMAS- 8 is a useful tool for measuring adherence among individuals with CF. This study supports the recommendations for annual screening of depression and anxiety in those with CF ages 12 years and older and highlights the mental health needs of young adults with $C F$ that need increased attention.

\section{Abbreviations}

CF: cystic fibrosis; HRQoL: health-related quality of life; TIDES: The International Depression/Anxiety Epidemiological Study of CF patients; HADS: The Hospital Anxiety and Depression scale; CES-D: Center for Epidemiological Studies Depression Scale; FEV : forced expiratory volume in one second; MMAS-8: Morisky Medication Adherence Scale; MDI: The Major Depression Inventory; CFQ-R: The Cystic Fibrosis Questionnaire-Revised-Teen/Adult version; CFRD: cystic fibrosis related diabetes.

\section{Authors' contributions}

KBK: study design, data collection and statistical analysis, drafting and manuscript writing. TP: study design, data collection and critical revision. LHM: assisted in statistical analysis, critical revision. MJ: advised on interpretation of results and critical revision. MS: study design, critical revision. ALQ: advised on study design, analytic plan, revision of the manuscript and contributed to the final version. TK: conception and design, data collection, critical revision. KAB: study design, manuscript writing, advised on interpretation on results, critical revision. All authors read and approved the final manuscript.

\section{Author details \\ ${ }^{1}$ The Department of Infectious Diseases, Copenhagen University Hospital, Rigshospitalet, Blegdamsvej 9, 2100 Copenhagen, Denmark. ${ }^{2}$ Cystic Fibrosis Center Copenhagen, Department of Pediatric and Adolescent Medicine, Copenhagen University Hospital, Rigshospitalet, Copenhagen, Denmark. ${ }^{3}$ The Department of Public Health, University of Copenhagen, Copenhagen, Den- mark. ${ }^{4}$ The University Hospital Center for Health Research (UCSF), Copenha- gen University Hospital, Rigshospitalet, Copenhagen, Denmark. ${ }^{5}$ Department of Psychology, University of Miami, Coral Gables, FL, USA. ${ }^{6}$ Center of Adoles- cent Medicine, Department of Pediatric and Adolescent Medicine, Copenha- gen University Hospital, Rigshospitalet, Copenhagen, Denmark.}

\section{Acknowledgements}

This was an investigator initiated study. The primary investigator (KBK) is grateful to have received an unconditional grant from Gilead Sciences Inc. and from the Finsen Center, Rigshospitalet.

\section{Competing interests}

The authors declare that they have no competing interests.

\section{Ethical approval and consent to participate}

All procedures were in accordance with the ethical standards of the institutional and/or national research committee and with the 1964 Declaration of Helsinki and its later amendments. This study was registered at the Danish Data Protection Agency (j.nr. 30-0918). In accordance with the regulations of the Scientific Ethics Review Committee System of Denmark, questionnairebased studies do not need ethical approval. Participants gave informed verbal consent. The questionnaires were provided with an explanatory letter indicating that a final consent was inferred from completing and returning the questionnaire.

Received: 14 April 2016 Accepted: 18 July 2016

Published online: 29 July 2016

\section{References}

Arnet I, Metaxas C, Walter PN, Morisky DE, Hersberger KE (2015) The 8-item Morisky Medication Adherence Scale translated in German and validated against objective and subjective polypharmacy adherence measures in cardiovascular patients. J Eval Clin Pract 21(2):271-277

Badlan K (2006) Young people living with cystic fibrosis: an insight into their subjective experience. Health Soc Care Community 14(3):264-270

Bech P, Rasmussen NA, Olsen LR, Noerholm V, Abildgaard W (2001) The sensitivity and specificity of the Major Depression Inventory, using the Present State Examination as the index of diagnostic validity. J Affect Disord 66(2-3):159-164

Bech P, Timmerby N, Martiny K, Lunde M, Soendergaard S (2015) Psychometric evaluation of the Major Depression Inventory (MDI) as depression severity scale using the LEAD (Longitudinal Expert Assessment of All Data) as index of validity. BMC Psychiatry 15:190

Bregnballe V, Thastum M, Lund LD, Hansen CR, Preissler T, Schiotz PO (2008) Validation of the Danish version of the revised cystic fibrosis quality of life questionnaire in adolescents and adults (CFQ-R14+). J Cyst Fibros 7(6):531-536

Bregnballe V, Schiotz PO, Boisen KA, Pressler T, Thastum M (2011) Barriers to adherence in adolescents and young adults with cystic fibrosis: a questionnaire study in young patients and their parents. Patient Prefer Adherence 5:507-515

Cameron IM, Crawford JR, Lawton K, Reid IC (2008) Psychometric comparison of PHQ-9 and HADS for measuring depression severity in primary care. $\mathrm{Br}$ J Gen Pract 58(546):32-36

Chick N, Meleis Al (1986) Transitions: a nursing concern. In: Nursing research methodology. Aspen Publication, Boulder, pp 237-257 Retrived at http:// repository.upenn.edu/nrs/9

Culig J, Leppee M (2014) From Morisky to Hill-bone; self-reports scales for measuring adherence to medication. Coll Antropol 38(1):55-62

Fidika A, Herle M, Goldbeck L (2014) Symptoms of depression impact the course of lung function in adolescents and adults with cystic fibrosis. BMC Pulm Med 14:205

Frangolias DD, Holloway CL, Vedal S, Wilcox PG (2003) Role of exercise and lung function in predicting work status in cystic fibrosis. Am J Respir Crit Care Med 167(2):150-157

George M, Rand-Giovannetti D, Eakin MN, Borrelli B, Zettler M, Riekert KA (2010) Perceptions of barriers and facilitators: self-management decisions by older adolescents and adults with CF. J Cyst Fibros 9(6):425-432

Goldbeck L, Fidika A, Herle M, Quittner AL (2014) Psychological interventions for individuals with cystic fibrosis and their families. Cochrane Database Syst Rev (6):CD003148. doi:10.1002/14651858.CD003148.pub3

Gross R, Bellamy SL, Chapman J, Han X, O'Duor J, Palmer SC et al (2013) Managed problem solving for antiretroviral therapy adherence: a randomized trial. JAMA Intern Med 173(4):300-306

Hankinson JL, Odencrantz JR, Fedan KB (1999) Spirometric reference values from a sample of the general US population. Am J Respir Crit Care Med 159(1):179-187

Havermans T, Colpaert K, Dupont LJ (2008) Quality of life in patients with cystic fibrosis: association with anxiety and depression. J Cyst Fibros 7(6):581-584

Jessup M, Parkinson C (2010) "All at sea": the experience of living with cystic fibrosis. Qual Health Res 20(3):352-364

Knudsen KB, Mathiesen ER, Eriksen V, Skov M, Nielsen KG, Johannesen J et al (2015) The development of diabetes among Danish cystic fibrosis patients over the last two decades. Pediatr Diabetes 16(3):219-226

Konstantinidis A, Martiny K, Bech P, Kasper S (2011) A comparison of the Major Depression Inventory (MDI) and the Beck Depression Inventory (BDI) in severely depressed patients. Int J Psychiatry Clin Pract 15(1):56-61

Lakens D (2013) Calculating and reporting effect sizes to facilitate cumulative science: a practical primer for t-tests and ANOVAs. Front Psychol 4:863 
MacKenzie T, Gifford AH, Sabadosa KA, Quinton HB, Knapp EA, Goss CH et al (2014) Longevity of patients with cystic fibrosis in 2000 to 2010 and beyond: survival analysis of the cystic fibrosis foundation patient registry. Ann Intern Med 161(4):233-241

Modi AC, Lim CS, Yu N, Geller D, Wagner MH, Quittner AL (2006) A multimethod assessment of treatment adherence for children with cystic fibrosis. J Cyst Fibros 5(3):177-185

Modi AC, Driscoll KA, Montag-Leifling K, Acton JD (2011) Screening for symptoms of depression and anxiety in adolescents and young adults with cystic fibrosis. Pediatr Pulmonol 46(2):153-159

Morisky DE, Ang A, Krousel-Wood M, Ward HJ (2008) Predictive validity of a medication adherence measure in an outpatient setting. J Clin Hypertens Greenwich Conn 10(5):348-354

O'Donohoe R, Fullen BM (2014) Adherence of subjects with cystic fibrosis to their home program: a systematic review. Respir Care 59(11):1731-1746

O'Sullivan BP, Freedman SD (2009) Cystic fibrosis. Lancet Lond Engl 373(9678):1891-1904

Olsen LR, Jensen DV, Noerholm V, Martiny K, Bech P (2003) The internal and external validity of the Major Depression Inventory in measuring severity of depressive states. Psychol Med 33(2):351-356

Quittner AL, Barker DH, Snell C, Grimley ME, Marciel K, Cruz I (2008) Prevalence and impact of depression in cystic fibrosis. Curr Opin Pulm Med 14(6):582-588

Quittner AL, Sawicki GS, McMullen A, Rasouliyan L, Pasta DJ, Yegin A et al (2012) Erratum to: psychometric evaluation of the Cystic Fibrosis Questionnaire-Revised in a national, US sample. Qual Life Res. 21(7):1279-1290

Quittner AL, Zhang J, Marynchenko M, Chopra PA, Signorovitch J, Yushkina Y et al (2014a) Pulmonary medication adherence and health-care use in cystic fibrosis. Chest 146(1):142-151

Quittner AL, Goldbeck L, Abbott J, Duff A, Lambrecht P, Sole A et al (2014b) Prevalence of depression and anxiety in patients with cystic fibrosis and parent caregivers: results of The International Depression Epidemiological Study across nine countries. Thorax 69(12):1090-1097
Quittner AL, Abbott J, Georgiopoulos AM, Goldbeck L, Smith B, Hempstead SE et al (2016) International Committee on Mental Health in Cystic Fibrosis: Cystic Fibrosis Foundation and European Cystic Fibrosis Society consensus statements for screening and treating depression and anxiety. Thorax 71(1):26-34

Ratjen F, Bell S, Rowe S, Goss C, Quittner A, Bush A (2015) Cystic fibrosis. Nat Rev Dis Primers (15010). doi:10.1038/nrdp.2015.10

Riekert KA, Bartlett SJ, Boyle MP, Krishnan JA, Rand CS (2007) The association between depression, lung function, and health-related quality of life among adults with cystic fibrosis. Chest 132(1):231-237

Sabaté E (2003) Adherence to long-term therapies-evidence for action. World Health Organization; Report no.: ISBN 9241545992

Sawicki GS, Goss CH (2015) Tackling the increasing complexity of CF care. Pediatr Pulmonol 50(Suppl 40):S74-S79

Sawicki GS, Rasouliyan L, McMullen AH, Wagener JS, McColley SA, Pasta DJ et al (2011) Longitudinal assessment of health-related quality of life in an observational cohort of patients with cystic fibrosis. Pediatr Pulmonol 46(1):36-44

Sawicki GS, Ren CL, Konstan MW, Millar SJ, Pasta DJ, Quittner AL (2013) Treatment complexity in cystic fibrosis: trends over time and associations with site-specific outcomes. J Cyst Fibros 12(5):461-467

Spoonhower KA, Davis PB (2016) Epidemiology of cystic fibrosis. Clin Chest Med 37(1):1-8

Targett K, Bourke S, Nash E, Murphy E, Ayres J, Devereux G (2014) Employment in adults with cystic fibrosis. Occup Med Oxf Engl 64(2):87-92

Yohannes AM, Willgoss TG, Fatoye FA, Dip MD, Webb K (2012) Relationship between anxiety, depression, and quality of life in adult patients with cystic fibrosis. Respir Care 57(4):550-556

\section{Submit your manuscript to a SpringerOpen ${ }^{\odot}$ journal and benefit from:}

- Convenient online submission

- Rigorous peer review

- Immediate publication on acceptance

- Open access: articles freely available online

- High visibility within the field

- Retaining the copyright to your article

Submit your next manuscript at springeropen.com 